\title{
Shortest Path Set Induced Vertex Ordering and its Application to Distributed Distance Optimal Formation Path Planning and Control on Graphs
}

\author{
Jingjin Yu Steven M. LaValle
}

\begin{abstract}
For the task of moving a group of indistinguishable agents on a connected graph with unit edge lengths into an arbitrary goal formation, it was shown that distance optimal paths can be computed to complete with a tight convergence time guarantee [30], using a fully centralized algorithm. In this study, we establish the existence of a more fundamental ordering of the vertices on the underlying graph network, induced by a fixed goal formation. The ordering leads to a simple distributed scheduling algorithm that assures the same convergence time. The vertex ordering also readily extends to more general graphs - those with arbitrary integer capacities and edge lengths - for which we again provide guarantees on the convergence time until the desired formation is achieved. Simulations, accessible via a web browser confirm our theoretical developments.
\end{abstract}

\section{INTRODUCTION}

For the task of moving a group of $n$ indistinguishable agents (or equivalently, robots or vehicles) on a connected graph with unit length edges into an arbitrary goal formation, an efficient centralized algorithm in [30] schedules all agents from an initial formation (configuration) to a goal formation, along paths with the smallest total distance. The authors further showed that, the schedule can be completed in $n+\ell-1$ time steps $(\ell$ is the longest distance between a pair of start and goal vertices), which is a tight bound.

In this paper, we significantly extend the previous results and show that, a directed acyclic graph (DAG) induced by the initial and goal formations admits an integral ordering of the vertices on the involved paths. The ordering, which may be used to compute the distance between any two vertices on a directed path of the DAG, is unique up to an additive constant. A simple algorithm based on this vertex ordering yields the same $n+\ell-1$ convergence time guarantee. This more fundamental structure provides a smooth transition from the problem formulation to the solution, which is missing from the constructive proof offered in [30].

Using this vertex ordering structure, once the initial agenttarget assignment is completed, the agents, via local (up to distance two) communication, can achieve the desired

Jingin $\mathrm{Yu}$ is with the Computer Science and Artificial Intelligence Laboratory, Massachusetts Institute of Technology, Cambridge, MA 02139 USA. E-mail: jingjin@csail.mit.edu. Steven M. LaValle is with the Department of Computer Science, University of Illinois at Urbana-Champaign, Urbana, IL 61801 USA. E-mail: lavalle@uiuc.edu. Acknowledgments. This work was supported in part by NSF grants 0904501 (IIS Robotics) and 1035345 (Cyberphysical Systems), MURI/ONR grant N00014-09-1-1052, and AFOSR grant FA95501210193. We thank the anonymous reviewers for their constructive comments that helped improve the quality of the final presentation of the materials.

${ }^{1}$ http://msl.cs.uiuc.edu/ jyul8/pe/distr-form.html. A Java plugin of version 6 or higher is required. formation, again in no more than $n+\ell-1$ time steps. To the best of our knowledge, this work provides the first multi-agent formation path planning algorithm that is both distance optimal and partially distributed, along with a tight convergence time guarantee. In general, global distance optimality is not achievable without direct or indirect global communication under our formulation 2 , implying that a fully distributed planning algorithm is not possible. As we will see, the ordering also allows easy extension of the results to graphs with edges having arbitrary integer lengths and nonunit capacities (i.e., more than one agent may be traveling on the same edge at a given instant).

When it comes to problems on formation, two subproblems come up. One of them is on the topic of formation control, which focuses on maintaining a formation of a group of vehicles; a desired formation, in these research, may be important for inter-vehicle communication or for maximizing certain utility functions [5, 24, 32]. Graph theoretic approaches are quite popular here, probably because vehicles and inter-vehicle constraints can be represented naturally with vertices and edges of graphs. The second sub-problem put more emphasis on how to achieve a desired formation via planning $[4,7,9,10,15,16,17,19,20,23,28,26,29,30]$, rather than to stabilize around a given formation. Among these, [15, 16, 17] appear to be mostly close to our effort in this paper (besides our earlier effort [30]). However, these works did not consider the issue of convergence time.

Generalizing the notion of formation to include multiple agents trying to agree on some common goal leads to the problem of consensus and rendezvous. This more general problem has remained a central research topic in control theory and robotics; see, e.g., [1, 2, 3, 6, , 8, 10, 12, 13, $14,18,21,22,24,25,27,31]$, to list a few. An early account of the rendezvous problem, as a form of formation control, appeared in [1], in which algorithmic solutions are provided for agents with limited range sensing capabilities. An $n$-dimensional rendezvous problem was approached via proximity graphs in [3]. For the consensus problem it is shown that averaging the behavior of close neighbors causes all agents to converge to the same behavior eventually [8]. We point out that, although this paper works with initial and goal vertex sets of $n$ distinct elements each, the presented results can be easily generalized to any number of goal

\footnotetext{
${ }^{2}$ A simple example: two agents occupy the diagonals of a square with two targets located on the other diagonal of the same square. Distance optimality is only possible if the two agents choose different targets before starting moving, which is not achievable without some form of global communication (direct or indirect).
} 
vertices between 1 and $n$, thus covering additional problems such as multi-agent rendezvous.

The rest of the paper is organized as follows. Section [II provides the problem formulation, an example, and its solution. Section [II] constructively proves the existence of the aforementioned vertex ordering on the induced DAG, followed by an application that schedules a set of distance optimal paths for the agents with a proven convergence time bound in Section IV] Section V then shows the scheduling algorithm can be easily turned into a distributed one, without relaxing the convergence time bound. We generalize the graph to have integer edge lengths and capacities in Section $\mathrm{VI}$ and conclude in Section VII.

\section{Formation Path Planning ON GRaphS}

Let $G=(V, E)$ be a connected, undirected, simple graph, in which $V=\left\{v_{i}\right\}$ is its vertex set and $E=\left\{\left(v_{i}, v_{j}\right)\right\}$ is its edge set. Let $A=\left\{a_{1}, \ldots, a_{n}\right\}$ be $n$ agents that move with unit speeds along the edges of $G$, with initial and goal vertices on $G$ specified by the injective maps $x_{I}, x_{G}: A \rightarrow V$, respectively. For convenience, $V, E$ also denote cardinalities of the sets $V, E$, respectively. Let $\sigma$ be a permutation that acts on the elements of $x_{G},\left(\sigma \circ x_{G}\right)$ is a map that defines a possible goal vertex assignment (a target formation).

A scheduled path is a map $p_{i}: \mathbb{Z}^{+} \rightarrow V$, in which $\mathbb{Z}^{+}:=$ $\mathbb{N} \cup\{0\}$. Intuitively, the domain of the paths is discrete time steps. A scheduled path $p_{i}$ is feasible for a single agent $a_{i}$ if it satisfies the following properties: (1) $p_{i}(0)=x_{I}\left(a_{i}\right),(2)$ for each $i$, there exists a smallest $k_{\min } \in \mathbb{Z}^{+}$such that $p_{i}\left(k_{\min }\right)=$ $\left(\sigma \circ x_{G}\right)\left(a_{i}\right)$ for some fixed $\sigma$ (i.e., same $\sigma$ for all $\left.1 \leq i \leq n\right)$ (that is, the end point of the path $p_{i}$ is some unique goal vertex), (3) for any $k \geq k_{\min }, p_{i}(k) \equiv\left(\sigma \circ x_{G}\right)\left(a_{i}\right)$, and (4) for any $0 \leq k<k_{\min },\left(p_{i}(k), p_{i}(k+1)\right) \in E$ or $p_{i}(k)=p_{i}(k+1)$.

We say that two paths $p_{i}, p_{j}$ are in collision if there exists $k \in \mathbb{Z}^{+}$such that $p_{i}(k)=p_{j}(k)$ (meet, or collision on a vertex) or $\left(p_{i}(k), p_{i}(k+1)\right)=\left(p_{j}(k+1), p_{j}(k)\right)$ (head-on, or collision on an edge). If $p(k)=p(k+1)$, the agent stays at vertex $p(k)$ between the time steps $k$ and $k+1$.

Problem 1 Given a 4-tuple $\left(G, A, x_{I}, x_{G}\right)$, find a set of paths $P=\left\{p_{1}, \ldots, p_{n}\right\}$ and a fixed $\sigma$ such that $p_{i}$ 's are feasible paths for respective agents $a_{i}$ 's for this $\sigma$ and no two paths $p_{i}, p_{j}$ are in collision.

Note that in the definition, we assume that edges of $G$ have unit lengths and capacities. That is, it takes unit time for an agent to cross an edge and no two agents can be on the same edge at the same time. This implicit assumption is used throughout Section $\Pi \mathrm{V}$ and relaxed in Section VI

To familiarize readers with the problem and its solution, look at the example in Fig. 11 The underlying graph $G$ is a $6 \times 7$ grid with holes. Assigning the top left corner coordinates $(0,0)$ and bottom right coordinates $(6,5)$, $x_{I}(A)=\{(0, i-1)\}, x_{G}(A)=\{(6, i-1)\}, 1 \leq i \leq 6$. That is, we want to move the agents from left to right. A solution to this problem that is distance optimal is given in Table [ corresponding to a schedule of the multi-colored paths in Fig.

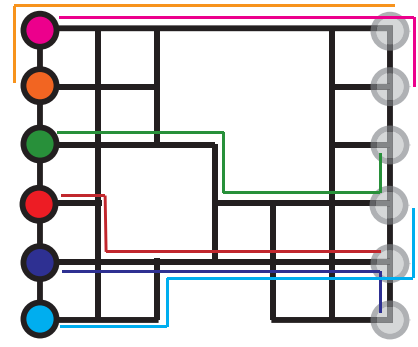

Fig. 1. A $6 \times 7$ grid with some vertices removed. The colored discs on the left represent the initial formation and the gray discs the goal formation. The colored paths represent the paths (not yet scheduled to avoid collision).

1. Here, distance optimality seeks to minimize the total path lengths of all agents. Each main entry of the table designates the coordinates of the vertex an agent should be staying at the given time step.

TABLE I

\begin{tabular}{clllllllll}
\hline \hline \multirow{2}{*}{ Agent } & \multicolumn{1}{c}{ Time Step } \\
\cline { 2 - 11 } & 0 & 1 & 2 & 3 & 4 & 5 & 6 & 7 & 8 \\
\hline 1 & 0,0 & 1,0 & 2,0 & 3,0 & 4,0 & 5,0 & 6,0 & 6,1 & 6,1 \\
\hline 2 & 0,1 & 0,0 & 1,0 & 2,0 & 3,0 & 4,0 & 5,0 & 6,0 & 6,0 \\
\hline 3 & 0,2 & 1,2 & 2,2 & 3,2 & 3,3 & 4,3 & 5,3 & 6,3 & 6,2 \\
\hline 4 & 0,3 & 1,3 & 1,4 & 1,4 & 2,4 & 3,4 & 4,4 & 5,4 & 6,4 \\
\hline 5 & 0,4 & 1,4 & 2,4 & 3,4 & 4,4 & 5,4 & 6,4 & 6,5 & 6,5 \\
\hline 6 & 0,5 & 1,5 & 2,5 & 2,4 & 3,4 & 4,4 & 5,4 & 6,4 & 6,3 \\
\hline \hline
\end{tabular}

\section{Formation INDUCED VERTEX ORDERING}

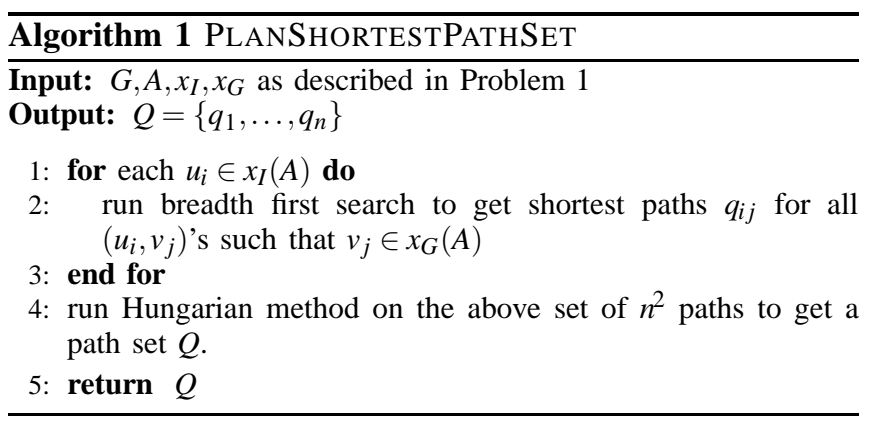

Given $x_{I}$ and $x_{G}$, it is relatively straightforward to obtain an unscheduled path set $Q=\left\{q_{1}, \ldots, q_{n}\right\}$ in which $q_{i}$ is a sequence of adjacent vertices (we use $Q$ to distinguish these paths from the scheduled paths, denoted $P$ ), with the help of the Hungarian method [11]. Our implementation is outlined in Algorithm 1 Let head $\left(q_{i}\right)$, $\operatorname{tail}\left(q_{i}\right)$, and $\operatorname{len}\left(q_{i}\right)$ denote the start vertex, end vertex, and length of $q_{i}$, respectively. The path set $Q$ returned from Algorithm 1 has several obvious properties, listed below

Property 2 For all $1 \leq i \leq n$, head $\left(q_{i}\right) \in x_{I}(A)$ and tail $\left(q_{i}\right) \in$ $x_{G}(A)$. For any two paths $q_{i}, q_{j}$, head $\left(q_{i}\right) \neq \operatorname{head}\left(q_{j}\right)$ and $\operatorname{tail}\left(q_{i}\right) \neq \operatorname{tail}\left(q_{j}\right)$.

\footnotetext{
${ }^{3}$ Properties 25 5 and Proposition 6 are from [30]; they are restated here to make this paper more self-contained.
} 
Property 3 Each path $q_{i}$ is a shortest path between head $\left(q_{i}\right)$ and $\operatorname{tail}\left(q_{i}\right)$ on $G$.

\section{Property 4 The total length of the path set $Q$ is minimal.}

Constructively guaranteed by Algorithm 1 Properties 2 and 3 ensure that the initial and goal vertices are paired up using shortest paths. Property 4 requires the total length of these paths to be minimal. From now on, $Q$ is always assumed to be a path set satisfying properties 2,4 It is not hard to see that Property 4 implies the following.

Property 5 If the edges of every path $q_{i} \in Q$ are oriented from head $\left(q_{i}\right)$ to tail $\left(q_{i}\right)$, no two paths share a common edge oriented in different directions.

Let $V(\cdot), E(\cdot)$ denote the vertex set and the undirected edge set of the input arguments, which can be either a path, $q_{i}$, or a set of paths, such as $Q$. We define an intersection between two paths as a maximal consecutive sequence of vertices and edges common to the two paths. Property 5 is a special case of a more general structure of the path set $Q$, stated in the following proposition.

Proposition 6 The path set $Q$ induces a directed acyclic graph $(D A G)$ structure on $E(Q)$.

Proposition 6 leads to a tight bound on the number of time steps to schedule the path set $Q$ [30]. Somewhat surprisingly, the DAG structure on $Q$ has an even stronger vertex ordering property that does not hold for DAGs in general; this is where the contribution of this paper starts. To state the property, we need some definitions for describing relationships between paths. Recall that two paths intersect (a symmetric relationship) if they share some common vertices or edges. Two paths $q_{i}, q_{j}$ are linked (again a symmetric relationship) if either $q_{i}, q_{j}$ intersect or both $q_{i}, q_{j}$ are linked to some $q_{k}$ (note that this is an inductive definition with a base case). A cluster $Q_{c}$ is a set of paths such that every pair of paths $q_{i}, q_{j} \in Q_{c}$ are linked. A path cluster $Q_{c}$ is a maximal cluster of $Q$ if $Q_{c}$ is a cluster and no other path $q_{i} \in Q \backslash Q_{c}$ is linked to a path $q_{j} \in Q_{c}$.

For each path $q_{i} \in Q$, a distance value function, $d_{i}$ : $V\left(q_{i}\right) \rightarrow \mathbb{Z}^{+}$, is defined as

$$
d_{i}(u)= \begin{cases}0 & u=h e a d\left(q_{i}\right), \\ \operatorname{dist}\left(\operatorname{head}\left(q_{i}\right), u\right) & \text { otherwise, }\end{cases}
$$

in which $\operatorname{dist}(u, v)$ denotes the shortest distance between $u, v$ on the graph $G$. Distance value functions can be defined similarly for an arbitrary set of vertices. Given the generalized definition, we say that one distance value function, $d^{\prime}$, respects another one, $d$, if $d^{\prime}$ is defined for all of $d^{\prime}$ 's domain and for any $u, v$ on which $d$ is defined,

$$
d^{\prime}(u)-d^{\prime}(v)=d(u)-d(v) .
$$

In an unscheduled path set $Q$, for any two paths $q_{i}, q_{j}$ that intersect, a distance value function can be constructed to respect both $d_{i}$ and $d_{j}$.
Lemma 7 If a vertex $u^{*}$ belongs to the intersection of two paths $q_{i}, q_{j} \in Q$, then the distance value function

$$
d_{c}(u)= \begin{cases}d_{i}(u) & u \in V\left(q_{i}\right), \\ d_{c}\left(u^{*}\right)+d_{j}(u)-d_{j}\left(u^{*}\right) & u \in V\left(q_{j}\right),\end{cases}
$$

respects both $d_{i}$ and $d_{j}$.

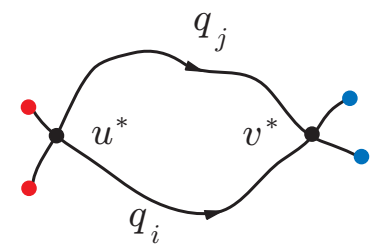

Fig. 2. Two intersections between two paths.

Proof. By definition, $d_{c}$ respects $d_{i}$. WLOG, let $u^{*}$ from (3) be the (unique) vertex on $q_{i}$ with the smallest $d_{i}$ value. If there is a single intersection (or common segment) between $q_{i}$ and $q_{j}$, then $d_{c}$ respects $d_{j}$ since by Property $5, q_{i}$ and $q_{j}$ cannot have edges oriented differently. If not, let $v^{*} \neq u^{*}$ be an arbitrary intersection point of $q_{i}$ and $q_{j}$ such that the segments of $q_{i}$ and $q_{j}$ between $u^{*}$ and $v^{*}$ differ by at least one vertex (see Fig. 2 one such configuration). Denote the segment between $u^{*}, v^{*}$ as $\omega_{i}$ and $\omega_{j}$ for $q_{i}$ and $q_{j}$, respectively, which must have the same orientation between $u^{*}$ and $v^{*}$ by Proposition 6 . We want to show that $d_{i}\left(v^{*}\right)=d_{c}\left(v^{*}\right)$, or

$$
\begin{array}{ll} 
& d_{i}\left(v^{*}\right)=d_{c}\left(v^{*}\right)=d_{c}\left(u^{*}\right)+d_{j}\left(v^{*}\right)-d_{j}\left(u^{*}\right) \\
& =d_{i}\left(u^{*}\right)+d_{j}\left(v^{*}\right)-d_{j}\left(u^{*}\right) \\
\Leftrightarrow \quad & d_{i}\left(v^{*}\right)-d_{i}\left(u^{*}\right)=d_{j}\left(v^{*}\right)-d_{j}\left(u^{*}\right) \\
\Leftrightarrow \quad & \operatorname{len}\left(\omega_{i}\right)=\operatorname{len}\left(\omega_{j}\right)
\end{array}
$$

If the last equation of (4) does not hold, without loss generality, we may assume that $\operatorname{len}\left(\omega_{i}\right)<\operatorname{len}\left(\omega_{j}\right)$; but then both paths should take $\omega_{i}$, a contradiction. Since $v^{*}$ is arbitrary, $d_{c}$ respects both $q_{i}$ and $q_{j}$.

We now show that (3) can be extended to a path cluster.

Theorem 8 Given a path cluster, $Q_{c}=\left\{q_{1}, \ldots, q_{m}\right\} \subset Q$, there exists a distance value function $d_{c}: V\left(Q_{c}\right) \rightarrow \mathbb{Z}^{+}$, such that $d_{c}$ respects $d_{i}$ for all $1 \leq i \leq m$.

PROOF. Lemma 7 proves the claim for any path cluster with no more than two paths. We inductively prove the claim for more than two paths. Assuming that a $d_{c}$ respects a (subcluster $Q_{c, k}=\left\{q_{1}, \ldots, q_{k-1}\right\} \subset Q_{c}, k \leq m-1$, we claim that the inductive definition

$d_{c}(u)= \begin{cases}d_{c}(u) & u \in V\left(\left\{q_{1}, \ldots, q_{k-1}\right\}\right), \\ d_{c}\left(u^{*}\right)+d_{k}(u)-d_{k}\left(u^{*}\right) & u \in V\left(q_{k}\right),\end{cases}$

extends $d_{c}$ so that it respects $d_{k}$ for a path $q_{k}$ that intersects paths in $\left\{q_{1}, \ldots, q_{k-1}\right\}$ at some vertex $u^{*}$. The claim trivially holds if $q_{k}$ intersects paths in $\left\{q_{1}, \ldots, q_{k-1}\right\}$ only once or $q_{k}$ intersects a single path $q_{i}, 1 \leq i \leq k-1$ more than once. 


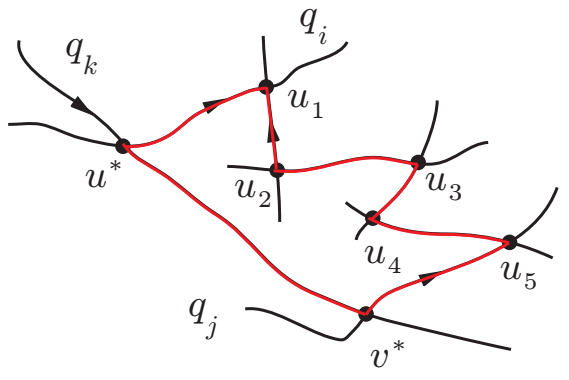

Fig. 3. A typical multiple path intersection.

The non-trivial case has $q_{k}$ intersect multiple paths in $Q_{c, k}$. WLOG, assume that $q_{k}$ intersects $q_{i}$ and $q_{j}, 1 \leq i, j \leq k-$ $1, i \neq j$, at $u^{*}$ and $v^{*}$, respectively (the order of $i, j$ does not matter), such that $v * \notin V\left(q_{i}\right)$ and $u^{*} \notin V\left(q_{j}\right)$ (otherwise it becomes the aforementioned trivial case). Additionally, we require that $u^{*}$ and $v^{*}$ have the two lowest (unique) $d_{k}$ values. We may then assume that the general structure of this setting is as illustrated in Fig. 3 (in the figure there are 7 paths on the undirected red cycle; there could be fewer or more paths). Note that single intersection points between paths are assumed due to Lemma 7 multiple intersections between the same pair of paths do not affect $d_{c}$ values.

We make the temporary assumption that the paths in Fig. 3 are oriented such that for any intersection point (for example $u^{*}$ ), the two involved paths (in the case of $u^{*}, q_{i}$ and $q_{k}$ ) take different orientations on the (red) cycle. With this assumption, on the undirected (red) cycle formed by intersecting paths, there is always an even number of paths between $u^{*}$ and $v^{*}$ besides $q_{k}$ (possibly after applying above path switching procedure many times). Let this even number be $2 b$ and the $2 b$ paths intersect at $u_{1}, \ldots, u_{2 b-1}$ (Fig. 3 shows the case where $b=3$ ). Also let $u^{*} \equiv u_{0}, v^{*} \equiv u_{2 b}$. To show that (5) extends $d_{c}$ to $d_{k}$, we need to show $d_{k}\left(v^{*}\right)-d_{k}\left(u^{*}\right)=$

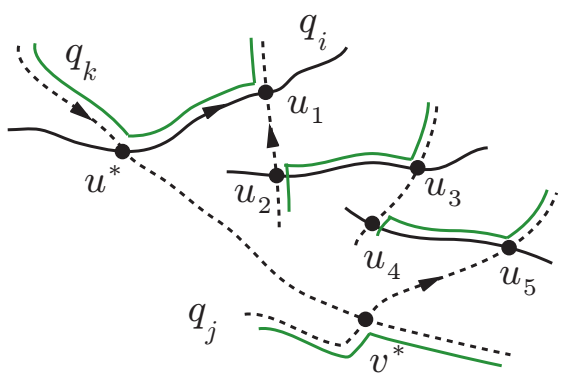

Fig. 4. Augmented paths. The dotted paths are the old paths, replaced by the green ones. Note that the two sets of paths (green and dotted ones) have the same initial and goal vertex sets.

$d_{c}\left(v^{*}\right)-d_{c}\left(u^{*}\right)$. If this is not true, WLOG, assume that $d_{k}\left(v^{*}\right)-d_{k}\left(u^{*}\right)>d_{c}\left(v^{*}\right)-d_{c}\left(u^{*}\right)$. For this case, we update segments of the paths as shown in Fig. 4. The update gives us a net gain of path length

$$
\begin{aligned}
& -\operatorname{dist}\left(u^{*}, v^{*}\right)+\sum_{i=0}^{b-1} \operatorname{dist}\left(u_{2 i}, u_{2 i+1}\right)-\sum_{i=1}^{b} \operatorname{dist}\left(u_{2 i-1}, u_{2 i}\right) \\
= & d_{k}\left(u^{*}\right)-d_{k}\left(v^{*}\right)+\sum_{i=0}^{b-1}\left(d_{c}\left(u_{2 i+1}\right)-d_{c}\left(u_{2 i}\right)\right) \\
& +\sum_{i=1}^{b}\left(d_{c}\left(u_{2 i-1}\right)-d_{c}\left(u_{2 i}\right)\right) \\
= & d_{k}\left(u^{*}\right)-d_{k}\left(v^{*}\right)+d_{c}\left(v^{*}\right)-d_{c}\left(u^{*}\right)<0,
\end{aligned}
$$

which contradicts Property 4. We conclude that (5) indeed extends $d_{c}$ to respect $d_{k}$.

If the temporary assumption does not hold (i.e., two intersecting paths take the same orientation on the cycle), the above proof holds via temporarily switching (a finite number of) paths. As an example, say segments of $q_{i}$ and $q_{k}$ take the same orientation on the red cycle. Then the new paths $q_{i}^{\prime}$ and $q_{k}^{\prime}$ given in Fig. 5 preserve the total path length. The rest of the proof (previously paragraphs) then applies with appropriate adjustments to how the paths are switched.

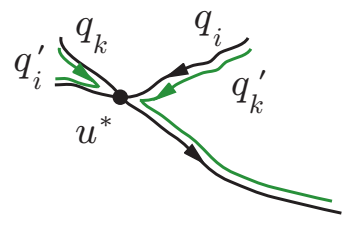

Fig. 5. A case in which $q_{i}$ has a different orientation compared to Fig. 3

We have so far shown that the $d_{c}$ respects $d_{k}$ on $q_{k}$ up to $v^{*}$. Inductively applying the same proof to other intersection points beyond $v^{*}$ proves the claim of the theorem.

\section{An Ordering-BASEd Scheduling Algorithm}

Assuming that a time optimal schedule seeks to minimize the time it takes the last agent to reach its goal, the following was established in [30].

Lemma 9 In general, distance optimality and time optimality for Problem 1$]$ cannot be simultaneously satisfied.

Furthermore, let $\ell$ be the largest pairwise distance between a member of $x_{I}(A)$ and a member of $x_{G}(A)$,

$$
\ell=\max _{\forall u \in x_{I}(A), v \in x_{G}(A)} \operatorname{dist}(u, v) .
$$

It was also shown in [30] that $n+\ell-1$ time steps is necessary to schedule a shortest path set $Q$ for an infinite family of instances of Problem 1. It was then shown that an unscheduled path set $Q$ can be turned into a scheduled path set $P$ with a maximum of $n+\ell-1$ time steps, providing a distance optimal schedule with a tight scheduling time bound. We now show that the vertex ordering induced by $x_{G}$ leads to a scheduling algorithm with the same guarantees on the scheduled paths' qualities. The new algorithm is simpler 
to implement and has a better running time of $O(n V \log n)$; it is not clear though, from a first look, that it should provide the said convergence time guarantee.

By Theorem 8 , each maximal path cluster $Q_{c} \subset Q$ can be assigned a distance value function $d_{c}$ that respects the distance function $d_{i}$ for each $q_{i} \in Q_{c}$. Since these individual $d_{c}$ 's have no common domain, they can be combined to give a global $d_{c}$ (for a fixed $Q$ ). Assuming such a $d_{c}$, which can be obtained easily using (5). Before scheduling the path set $Q$, we introduce a subroutine to handle the scenario illustrated in Fig. 6. In the figure, $Q=\left\{q_{1}, q_{2}\right\}$ with $\operatorname{head}\left(q_{i}\right)=u_{i}, \operatorname{tail}\left(q_{i}\right)=v_{i}$ for $i=1,2$. This path set cannot be scheduled as is, since $q_{1}$ is in the way of $q_{2}$. However, as

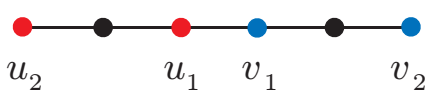

Fig. 6. A path set $Q$ that cannot be scheduled without modification.

agent $a_{1}$ reaches $v_{1}$, we can dynamically switch the goals of $q_{1}, q_{2}$. Note that the path set after this update still satisfies Properties 2, 4, For paths $q_{i}, q_{j}$, denote this path switching subroutine $\operatorname{switch}\left(q_{i}, q_{j}\right)$.

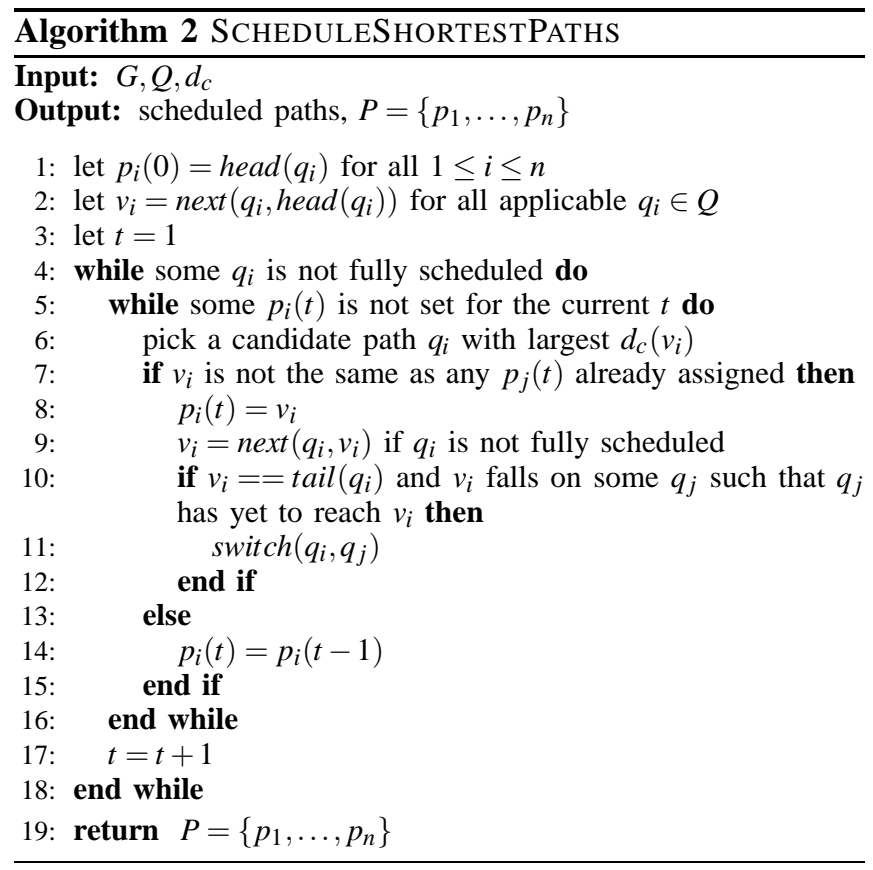

The path scheduling subroutine is outlined in Algorithm 2] in which the routine next $\left(q_{i}, v\right)$ returns the next vertex of path $q_{i}$ after vertex $v$. A path $q_{i}$ is fully scheduled if tail $\left(q_{i}\right)$ is assigned to $p_{i}(t)$ for some $t$. The scheduling routine never considers two paths $q_{i}, q_{j}$ running in opposite directions since Property 5 excludes such cases. Essentially, the scheduling algorithm let all paths from $Q$ take their respective courses simultaneously. Whenever two paths are competing for going to the same vertex, an arbitrary path is picked to go and the other one to stay put. With the switch $(\cdot, \cdot)$ subroutine to guarantee that no deadlock can occur, it is straightforward to see that the process must converge since at each $t$, at least one agent will make progress toward its goal. That is,

\section{Proposition 10 Algorithm 2 terminates in finite time.}

Denote the total path length of $Q$ as $\ell_{Q}$, then the convergence time (the time it takes for the formation to be completed) is no more than $\ell_{Q}$. However, as we have mentioned, Algorithm 2 provides a much stronger guarantee, as Theorem 11 will show. we apologize for the somewhat long proof but it seems more appropriate to have a long proof in this case than to split it into lemmas.

Theorem 11 Algorithm 2 provides a schedule that takes at most $n+\ell-1$ time steps to complete.

PROOF. We constructively prove the theorem starting with a path set $Q$. Let $Q_{c}=\left\{q_{1}, \ldots, q_{m}\right\}$ be an arbitrary maximal cluster of $Q$, it is clear that we only need to prove the claim for $Q_{c}$. Moreover, we only need to prove the bound for the special case in which the routine $\operatorname{switch}(\cdot, \cdot)$ is never invoked, since we can effectively consider the "dynamic switching" all happen at time step $t=0$.

We want to schedule all agents, $a_{1}, \ldots, a_{m}$, along $q_{1}, \ldots, q_{m}$, respectively, starting at $t=0$. Before starting, we create a list of numbers, $H$, indexed by possible $d_{c}$ values (as constructed in the proof of Theorem 8 in decreasing order. Since the cluster $Q_{c}$ is finite, $H$ is also finite. An entry of this list, $h_{d}$, is the number of agents whose current locations have a $d_{c}$ value of $d$. A list $H$ may look like

$$
\begin{array}{llllllllrrrrr}
d & : & 5 & 4 & 3 & 2 & 1 & 0 & -1 & -2 & -3 & -4 & \ldots \\
h_{d} & : & 0 & 0 & 3 & 0 & 2 & 1 & 0 & 0 & 0 & 2 & \ldots
\end{array}
$$

We note that for a fixed time step $t$, the only importance of the index $d$ in list $H$ is that it specifies the relative order of agents. In the above $H$, for example, $h_{3}=3$, as the first non-zero entry, means that there are 3 agents as the "front runners", followed by next non-zero entry $h_{1}=1$, suggesting that there is 1 agent two steps behind. From this observation, we may negate the index $d$ and at each time step $t$, align $h_{1}$ with the first non-zero entry of $H$. The above $H$ then becomes

$$
\begin{array}{llrlllllllll}
d & : & -1 & 0 & 1 & 2 & 3 & 4 & 5 & 6 & 7 & 8 \\
h_{d} & : & 0 & 0 & 3 & 0 & 1 & 2 & 0 & 0 & 0 & 2
\end{array}
$$

We can also remove the leading (and trailing) zero entries

$$
\begin{array}{llllllllll}
d & : & 1 & 2 & 3 & 4 & 5 & 6 & 7 & 8 \\
h_{d} & : & 3 & 0 & 1 & 2 & 0 & 0 & 0 & 2
\end{array}
$$

At this point, we partition the list into one or more sublists as follows. Starting at $d=0$, we look at the sum of first $k$ terms of $H$,

$$
S_{k}=\sum_{d=1}^{k} h_{d} .
$$

If it ever happens for some $k$, starting at 1 , that $S_{k}=k$, we group these $k$ terms of $H$ into a sublist and work with it. We 
call these sublists contiguous sublists. Applying the partition procedure to $H$ above, the first contiguous sublist, $H^{\prime}$, is

$$
\begin{array}{llllllll}
d & : & 1 & 2 & 3 & 4 & 5 & 6 \\
h_{d}^{\prime} & : & 3 & 0 & 1 & 2 & 0 & 0
\end{array}
$$

Now let us consider how the sublist may change after we let all agents start moving towards their respective goals. For the first group of 3 agents, at least one of them can move one step closer to its goal and at most two of them may not make any progress. The worst case happens in a situation illustrated in Fig. 7 When conflict like this happens, we pick a random agent to advance. Suppose the worst case happens, we update to have $h_{0}^{\prime}=1, h_{1}^{\prime}=2$.

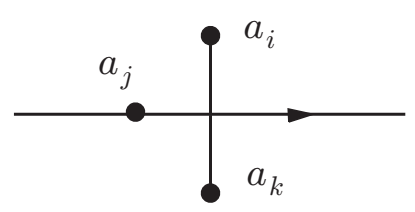

Fig. 7. A three-way conflict between three agents, in which case we pick any agent to go ahead and let the other two wait.

For the rest of the non-zero entries of $H^{\prime}$, if it is preceded by a zero entry, it means that there are no agents with $d_{c}$ values exactly one larger than this group of agents. Hence, at least one agent from this group of agents can make one step progress towards its goal. When this is applied to $h_{3}^{\prime}$, which has a value 1 , we update the sublist entries as $h_{2}^{\prime}=1, h_{3}^{\prime}=0$. After these two steps, the sublist $H^{\prime}$, still being processed between time steps, is

$$
\begin{array}{lllllllll}
d & : & 0 & 1 & 2 & 3 & 4 & 5 & 6 \\
h_{d}^{\prime} & : & 1 & 2 & 1 & 0 & 2 & 0 & 0
\end{array}
$$

Observe that the next non-zero entry, $h_{4}^{\prime}=2$, now has a preceding zero entry. Assume that only one agent advances, we update the entries to $h_{3}^{\prime}=1, h_{4}^{\prime}=1$. At the end of $t=1$, the updates give us $H^{\prime}$ as

$$
\begin{array}{lllllllll}
d & : & 0 & 1 & 2 & 3 & 4 & 5 & 6 \\
h_{d}^{\prime} & : & 1 & 2 & 1 & 1 & 1 & 0 & 0
\end{array}
$$

We can adjust $d$ to get

$$
\begin{array}{llllllll}
d & : & 1 & 2 & 3 & 4 & 5 & 6 \\
h_{d}^{\prime} & : & 1 & 2 & 1 & 1 & 1 & 0
\end{array}
$$

What does the entries of $H^{\prime}$ mean after each update? The entry $h_{1}^{\prime}$ represents the number of agents (paths) that never waited for others. Similarly, the entry $h_{i}^{\prime}$ represents the number of agents (paths) that never waited for more than $i-1$ steps. Note that the leading entry is $h_{1}^{\prime}=1$. Whenever the leading entry becomes 1 , the associated agent/path can no longer have any conflict with any other agent/path. That is, it has no more interaction with the rest of the agents. We claim that throughout the updates, at least $i$ agents never waited more than $i-1$ steps, which is easily verifiable via induction (we omit the details due to its length and irrelevance to the rest of the paper). The worst case happens when $H^{\prime}$ becomes all 1's as

$$
\begin{array}{llllllll}
d & : & 1 & 2 & 3 & 4 & 5 & 6 \\
h_{d}^{\prime} & : & 1 & 1 & 1 & 1 & 1 & 1
\end{array}
$$

In any case, by the above claim, agents from a contiguous sublist cannot "spill over" to the next contiguous list. If we apply what we have done to every contiguous sublist, the claim that at least $i$ agents never waited more than $i-1$ steps holds for all agents moving on the cluster $Q_{c}$. Since no agent travels a length more than $\ell$, We have proved the claim of the theorem.

\section{A Distributed Scheduling Algorithm}

From the constructive proof of Theorem 11 it is clear that within each maximal path cluster, an agent only needs to be aware of its neighbors within a distance of 2 to take appropriate actions. This implies that once agent-target assignment is done, global coordination is not required to schedule these agents, yielding partially distributed scheduling algorithm. Since local communication is often more reliable and easy to implement, such a scheduling algorithm is more desirable in general. In this section, we provide a local communication protocol which leads to a distributed scheduling algorithm, again with a convergence time of $n+\ell-1$. A common clock is assumed. We omit the pseudocode since it is a straightforward modification of Algorithm 2.

Assuming each agent is assigned a path, we will schedule them along these paths and possibly update their goals (targets) on the fly. Recall that by Property 5, we only need to worry about two agents occupying the same vertex at a given time step. This splits into two cases: (1) two agents want to move to the same vertex in one time step, and (2) one agent moves to a vertex while another agent is staying there. We now give a communication protocol, including a forward communication phase and a backward communication phase at each time step, that handles both cases.

Schedule 12 (Distributed Transfer Schedule) Repeat the following two communication phases until the desired formation is complete.

Forward communication phase. Assume that an agent $a_{i}$ is located on $v_{i}$ and wants to move to $v_{i+1}$. Agent $a_{i}$ first checks whether $v_{i+1}$ is occupied by some other agent $a_{j}$ and if it is, notifies $a_{j}$ of its intention and waits for $a_{j}$ 's response. At this point, $a_{j}$ will check whether it is already at its goal and if it is, switch its goal with $a_{i}\left(a_{j}\right.$ will also redo its forward communication phase if it already did). If no agent is occupying $v_{i+1}, a_{i}$ then looks for agents that also want to go to $v_{i+1}$. If there are, one agent is randomly picked to go to $v_{i+1}$ in the next time step. Alternatively, we could deterministically pick an agent (e.g. based on identities of the vertices occupied by the agents). Other agents wanting to go to $v_{i+1}$ then must wait one time step. Since we are dealing a finite number of agents and there are no cycles on a DAG, the forward communication phase will stop after at most $O(n)$ messages, each with a size of $O(\log V)$. 
Backward communication phase. Next, an agent that has received requests from a following agent needs to respond back. Let such two adjacent agents be $a_{i}$ and $a_{j}$, occupying $v_{i}, v_{i+1}$, respectively, with $a_{i}$ wanting to go to $v_{i+1}$. There are two sub-cases. In the first sub-case, $a_{j}$ moves and notifies $a_{i}$ that it may go ahead and move to $v_{i+1}$. If $a_{j}$ gets multiple requests to occupy $v_{i+1}$ then a randomly agent is selected to proceed (again, this can be made deterministic). In the second sub-case, $a_{j}$ cannot move because another agent tells it so. It then simply relay that message backward. Clearly, the backward communication will stop after at most $O(n)$ messages, each with a size of $O(\log V)$.

Schedule 12 has a similar algorithmic complexity compared with the centralized version. Time wise, we have

Corollary 13 Schedule 12 transfers all agents to achieve the desired formation in $O(n+\ell-1)$ time steps.

PROOF SKetch. The only difference introduced by the distributed schedule is the possibility that an agent $a_{j}$ needs to move to some agent $a_{i}$ 's goal vertex $v_{i}$, which is already occupied by $a_{i}$. In this case, we switch the goals of $a_{i}$ and $a_{j}$ (and the associated paths of $a_{i}$ and $a_{j}$ ). This switch has the net effect of delaying $a_{i}$ by one time step and speed up $a_{j}$ by one time step. The delay does not make $a_{i}$ reach its new goal later than $a_{j}$ so the switch has no negative effect on the total convergence time; the proof technique from Theorem 11 then applies with some modification. Note that we need to work with an entire path cluster in this case.

The scheduling algorithm is fairly simple to implement, as we did in a Java simulation (see abstract for the link). A snapshot of a running session is provided in Fig. 8 We do not provide computational evaluation here since the overall algorithm has similar running time as the algorithm from [30]. Readers interested in computational time on large instances may refer to [30] for more details.

VI. INTEGER EDGE LENGTHS AND CAPACITIES

So far we have assumed that we work with a graph $G$ with unit edge lengths and capacities. That is, an edge takes a unit of time to cross and can hold one agent at a time. We now relax this assumption to allow non-unit edge lengths and capacities. Formally, let $d, c: E \rightarrow \mathbb{Z}^{+}$be the edge length map and edge capacity map, respectively. We assume that for any $e \in E, d(e) \geq c(e)$, which is generally true for physical robots with non-negligible sizes (up to a multiplicative constant). The main goal of this section is to extend the results from previous sections under this setup. Note that the definition of scheduled paths and feasible paths from Section II need to be updated since it may take multiple time steps for an agent to cross an edge. Thus, a scheduled path $p_{i}$ becomes a partial map as it may be undefined for some time steps. We omit formal descriptions of these required updates since they are intuitive but lengthy to state.

It is clear that Algorithm 1 is insensitive to edge length. Therefore, the algorithm again produces an unscheduled path set $Q$ satisfying Properties 2,5. Moreover, all results from

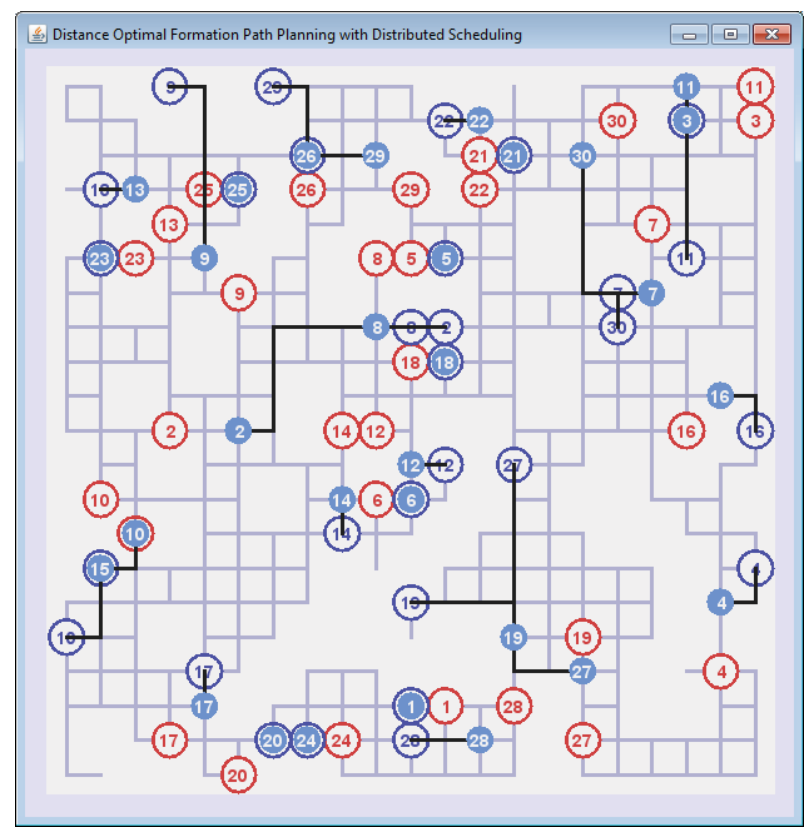

Fig. 8. A simulation capture. The red/blue circles and numbers are the start/goal locations (already assigned to have shortest total distance). The light blue solid discs represent the agents. The bold black lines are the paths yet to be completed.

Section III continue to hold with edge lengths that are not all ones. On the other hand, scheduling the path set $Q$ becomes slightly trickier, since depending on edge capacities, one or more agent may be on the same edge during within one time step. To simplify the analysis, we look at two extreme cases: (1) for all $e \in E, c(e)=d(e)$, and (2) for all $e \in E, c(e) \equiv 1$. The first case models scenarios that allow bumper to bumper road traffic. This case is easy to handle, due to the following observation: By subdividing each edge $e \in E$ into $d(e)$ edges of unit length, we obtain a new graph $G$ with unit edge length and capacity. We turn our attention to the second case, which models bottleneck edges such as a long and thin bridge. First we establish a lower bound.

Lemma 14 Assume $\forall e \in E, c(e) \equiv 1$ and let $d_{\max }=$ $\max _{e \in E} d(e)$. Then $\ell+(n-1) d_{\max }$ time steps is necessary to schedule $n$ agents along a shortest path set $Q$.

Proof. In the instance of Problem 1 shown in Fig. 9 assume that all edges have the same length $d$; hence, $d_{\max }=d$. The graph $G$ is two stars with their centers connected by a single path; the red vertices form $x_{I}(A)$ and the blue ones $x_{G}(A)$. It is clear that all red vertices are of distance $\ell$ to all blue vertices. Given this problem instance, all agents must go through the path $u v \ldots x y$ sequentially. To optimize arrival time, the first agent (say $a_{1}$ ) to reach goal must visit $u$ at $t=d_{\max }$. Consequently, $a_{1}$ cannot reach $v$ earlier than $t=2 d_{\max }$. This implies that no other agent can head to $v$ from $u$ before $t=2 d_{\max }$, due to the unit edge capacity constraint. Via simple induction, the last agent arriving at $u$ cannot leave it before $t=n d_{\text {max }}$. Therefore, it cannot arrive earlier than $t=\ell+(n-1) d_{\max }$. 


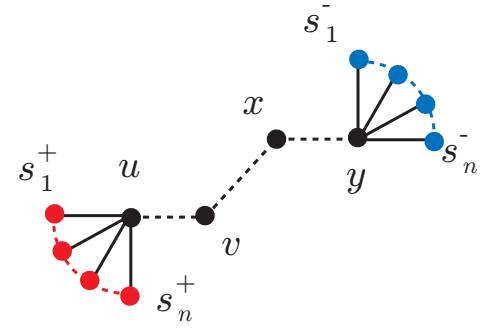

Fig. 9. An instance of Problem 1 for demonstrating the necessity claim of Lemma 14

If we pretend that all edges have the same length $d_{\max }$, Algorithm 2 can be easily extended to schedule a shortest path set $Q$. Clearly, this provides an overestimate of the total time it takes to schedule $Q$. Since no agent is delayed more than $(n-1) d_{\max }$ time steps, the following corollary to Theorem 11 is immediate.

Corollary 15 Assume $\forall e \in E, c(e) \equiv 1$ and let $d_{\max }=$ $\max _{e \in E} d(e)$. Algorithm 2 schedules a shortest path set $Q$ such that the scheduled path set requires at most $\ell+(n-$ 1) $d_{\max }$ time steps to complete.

Thus, the time bound $\ell+(n-1) d_{\max }$ is tight for the unit edge capacity case. Combining the two extreme cases together, we have the following conclusion.

Theorem 16 For the extension of Problem 1 with integer edge lengths and capacities in which $1 \leq c(e) \leq d(e)$ for all $e \in E$, the time bound $\ell+(n-1) d_{\max }$ is sufficient and necessary to schedule $n$ agents along a shortest path set $Q$.

Straightforward complexity analysis shows that for integer edge lengths and capacities, the running time of the entire algorithm becomes $O\left(n V^{2}+n V d_{\max }\right)$.

\section{CONCLUSION AND FUtURE WORK}

In this paper, for the multi-agent formation path planning problem on graphs, we showed the existence of a vertex ordering structure induced by the initial and goal formations, which in turn admits a simple and natural scheduling algorithm for coordinating the shortest paths amongst the indistinguishable agents with a tight convergence time guarantee. Furthermore, the ordering allows the scheduling algorithm to be distributed. We then showed that the ordering as well as the convergence time guarantee generalize to integer edge lengths and capacities.

Seeing how the vertex ordering helped us in obtaining a distributed scheduling algorithm without sacrificing convergence time, we plan to study further implications of this order structure. On the practical side, we hope to put the algorithm onto robots to test its performance in real world applications. With increased availability of cheap and fast wireless communication capabilities, we believe our algorithm can be used on formation control problems for a large group of robots or other types of vehicles in practice.

\section{REFERENCES}

[1] H. Ando, Y. Oasa, I. Suzuki, and M. Yamashita. Distributed memoryless point convergence algorithm for mobile robots with limited visibility. IEEE Transactions on Automatic Control, 15(5):818-828, October 1999.

[2] T. Balch and R. C. Arkin. Behavior-based formation control for multirobot teams. IEEE Transactions on Robotics \& Automation, 14(6):926-939, 1998

[3] J. Cortés, S. Martínez, and F. Bullo. Robust rendezvous for mobile autonomous agents via proximity graphs in arbitrary dimensions. IEEE Transactions on Automatic Control, 51(8):1289-1298, August 2006.

[4] M. A. Erdmann and T. Lozano-Pérez. On multiple moving objects. In Proceedings IEEE International Conference on Robotics \& Automation, pages 1419-1424, 1986.

[5] J. A. Fax and R. M. Murray. Information flow and cooperative control of vehicle formations. IEEE Transactions on Automatic Control, 49(9):1465-1476, September 2004.

[6] V. Gazi. Stability of a discrete-time asynchronous swarm with timedependent communication links. IEEE Transactions on Systems, Man, and Cybernetics: Part B, 38(1):267-274, February 2008.

[7] Y. Guo and L. E. Parker. A distributed and optimal motion planning approach for multiple mobile robots. In Proceedings IEEE International Conference on Robotics and Automation, pages 2612-2619, 2002.

[8] A. Jadbabaie, J. Lin, and A. S. Morse. Coordination of groups of mobile autonomous agents using nearest neighbor rules. IEEE Transactions on Automatic Control, 48(6):988-1001, 2003.

[9] K. Kant and S. Zucker. Towards efficient trajectory planning: The path velocity decomposition. International Journal of Robotics Research, 5(3):72-89, 1986

[10] S. Kloder and S. Hutchinson. Path planning for permutation-invariant multirobot formations. IEEE Transactions on Robotics, 22(4):650665, 2006.

[11] H. W. Kuhn. The Hungarian method for the assignment problem. Naval Research Logistics Quarterly, 2:83-97, 1955.

[12] J. Lin, A. S. Morse, and B. D. O. Anderson. The multi-agent rendezvous problem. part 1: The synchronous case. SIAM Journal on Control and Optimization, 46(6):2096-2119, November 2007.

[13] J. Lin, A. S. Morse, and B. D. O. Anderson. The multi-agent rendezvous problem. part 2: The asynchronous case. SIAM Journal on Control and Optimization, 46(6):2120-2147, November 2007.

[14] Z. Lin, M. Broucke, and B. Francis. Local control strategies for groups of mobile autonomous agents. IEEE Transactions on Automatic Control, 49(4):622-629, April 2004.

[15] L. Liu and D. A. Shell. Large-scale multi-robot task allocation via dynamic partitioning and distribution. Autonomous Robots, 33(3):291307, 2012.

[16] L. Liu and D. A. Shell. Tunable routing solutions for multi-robot navigation via the assignment problem: A $3 \mathrm{~d}$ representation of the matching graph. In Proceedings IEEE International Conference on Robotics \& Automation, pages 4800-4805, 2012.

[17] D. Miklic, S. Bogdan, R. Fierro, and S. Nestic. A discrete grid abstraction for formation control in the presence of obstacles. In Proceedings IEEE/RSJ International Conference on Intelligent Robots and Systems, pages 3750-3755, 2009.

[18] L. Moreau. Stability of multiagent systems with time-dependent communication links. IEEE Transactions on Automatic Control, 50(2):169-182, February 2005

[19] P. A. O’Donnell and T. Lozano-Pérez. Deadlock-free and collisionfree coordination of two robot manipulators. In Proceedings IEEE International Conference on Robotics \& Automation, pages 484-489, 1989.

[20] J. Peng and S. Akella. Coordinating multiple robots with kinodynamic constraints along specified paths. In J.-D. Boissonat, J. Burdick, K. Goldberg, and S. Hutchinson, editors, Algorithmic Foundations of Robotics V (WAFR 2002), pages 221-237. Springer-Verlag, Berlin, 2002.

[21] W. Ren and R. W. Beard. Consensus seeking in multi-agent systems under dynamically changing interaction topologies. IEEE Transactions on Automatic Control, 50(5):655-661, May 2005.

[22] B. Shucker, T. Murphey, and J. K. Bennett. Switching rules for decentralized control with simple control laws. In American Control Conference, pages 1485-1492, Jul 2007. 
[23] T. Siméon, S. Leroy, and J.-P. Laumond. Path coordination for multiple mobile robots: A resolution complete algorithm. IEEE Transactions on Robotics \& Automation, 18(1):42-49, February 2002.

[24] B. Smith, M. Egerstedt, and A. Howard. Automatic generation of persistent formations for multi-agent networks under range constraints. ACM/Springer Mobile Networks and Applications Journal, 14(3):322335, June 2009.

[25] S. L. Smith, M. E. Broucke, and B. A. Francis. Curve shortening and the rendezvous problem for mobile autonomous robots. IEEE Transactions on Automatic Control, 52(6):1154-1159, June 2007.

[26] D. M. Stipanovic, S. Shankaran, and C. J. Tomlin. Multi-agent avoidance control using an m-matrix property. Electronic Journal of Linear Algebra, 12:64-72, May 2005.

[27] H. Tanner, G. Pappas, and V. Kumar. Leader-to-formation stability. IEEE Transactions on Robotics \& Automation, 20(3):443-455, Jun 2004.

[28] J. van den Berg, J. Snoeyink, M. Lin, and D. Manocha. Centralized path planning for multiple robots: Optimal decoupling into sequential plans. In Proceedings Robotics: Science and Systems, 2009.

[29] P. Švestka and M. H. Overmars. Coordinated path planning for multiple robots. Robotics and Autonomous Systems, 23(3):125-152, 1998.

[30] J. Yu and S. M. LaValle. Distance optimal formation control on graphs with a tight convergence time guarantee. In Proceedings IEEE Conference on Decision \& Control, pages 4023-4028, 2012.

[31] J. Yu, S. M. LaValle, and D. Liberzon. Rendezvous without coordinates. IEEE Transactions on Automatic Control, 57(2):421-434, February 2012.

[32] M. M. Zavlanos, M. B. Egerstedt, and G. J. Pappas. Graph-theoretic connectivity control of mobile robot networks. Proceedings of the IEEE, 99(9):1525-1540, September 2011. 